\title{
Active Tunable THz Metamaterial Array Implemented in CMOS Technology
}

Yongshan Liu ${ }^{1,2,3 \dagger}$, Tong Sun ${ }^{1,2 \dagger}$, Xiaojun Wü*, Zhongyang Bai ${ }^{4}$, Yun Sun ${ }^{1,3}$, Helin Li ${ }^{1,2}$, Haoyi Zhang ${ }^{1}$, Kanglong Chen ${ }^{4}$, Cunjun Ruan ${ }^{4}, Y u z u$ Sun ${ }^{1,2}$, Yuanqi Hu ${ }^{1,2,3}$, Tianxiao Nie $^{1,2,3} *$ Lianggong Wen $n^{1,2,3} *$

${ }^{1}$ School of Microelectronics, Beihang University, Beijing, 100191, China.

${ }^{2}$ Beihang-Goertek Joint Microelectronics Institute, Qingdao Research Institute, Beihang University, Qingdao, 266000, China.

${ }^{3}$ Hefei Innovation Research Institute, Beihang University, Hefei 230013, China.

${ }^{4}$ School of Electronics and Information Engineering, Beihang University, Beijing 100191, China.

†These authors contributed equally to this paper.

*Correspondence:

Lianggong Wen_Email: wenlg@buaa.edu.cn

Tianxiao Nie Email: nietianxiao@buaa.edu.cn

Xiaojun Wu Email: xiaojunwu@buaa.edu.cn

\section{Abstract}

Terahertz (THz) metamaterial modulators offer multifaceted capabilities for various practical applications such as $\mathrm{THz}$ imaging, wireless communications, sensing, et cetera. However, compared to the modulation devices for other electromagnetic bands, the ubiquitous proliferation of $\mathrm{THz}$ applications is severely impeded by the tremendous lack of complementary metal-oxide-semiconductor (CMOS) compatible technology. Here we demonstrate a CMOS based actively tunable THz metamaterial array (C-ATTMA) with split ring resonators (SRRs). The THz metamaterial modulators can be externally controlled with an electrically-controlled dynamic. The C-ATTMA fabricated by the $180 \mathrm{~nm}$ CMOS technology featuring a resonant frequency of $0.30 \mathrm{THz}$ was connected to the source and drain of a bottom metal-oxidesemiconductor field effect transistor (MOSFET) through the vias. By delicately controlling the MOSFET gate voltage, the equivalent circuit response of the C-ATTMA was actively engineered, enabling tailoring $\mathrm{THz}$ resonance frequencies. Under a gate voltage of $1.8 \mathrm{~V}$, we 
successfully realized a $35 \mathrm{GHz}$ modulation bandwidth with $3^{\circ}$ phase modulation. The exhibited CMOS-compatible electrically-regulated $\mathrm{THz}$ metamaterials may provide enormous potentials for implementing $\mathrm{THz}$ wireless communications, information encryption, $\mathrm{THz}$ compressed sensing imaging, et cetera.

Keywords: $\mathrm{THz}$ radiation, active tuning metamaterial, CMOS compatible, frequency modulation, phase control.

\section{Introduction}

Terahertz (THz) electromagnetic band, which ranges from $100 \mathrm{GHz}$ or $300 \mathrm{GHz}$ to $10 \mathrm{THz}$ [13], has received widespread attention in recent decades due to the unique outstanding properties and potential applications, such as line-of-sight telecommunications $[4,5]$, sensing [6-8] and imaging [9]Error! Reference source not found.. Especially the rapid development in effective THz sources, such as quantum cascade lasers [10, 11], uni-traveling-carrier photodiodes [12, 13] and spintronic $\mathrm{THz}$ emitters [14-18] has accelerated $\mathrm{THz}$ researches moving from laboratory to real applications. On the other hand, functional devices such as $\mathrm{THz}$ modulators play an essential role for accelerating the THz devices' proliferations. However, conventional THz modulations are based on optical [19], mechanical [20], and heat stimulus [21], limited by the intrinsic properties of the material $[22,23]$. Intriguingly, metamaterials based modulators enable electromagnetic wave manipulation, featuring artificially designable structures and abundant external controllability [24].

Currently, most metamaterials adopt static modulation methods and have demonstrated powerful modulation effect [24-27]. However, static metamaterials face the disadvantage that once fabricated, the modulation performance is statically decided. In order to solve this problem, various materials and devices were applied to the metamaterial structure for active tuning of the metamaterial devices [28-30]. The photosensitive metamaterial planar arrays were fabricated on light-sensitive material substrates. The response of the photosensitive 
metamaterials can be controlled by external light pumping, and the free carriers in the substrate, such as GaAs [31] and photoactive silicon [19] can be excited. Such modulation method is very promising for all-optical controlled $\mathrm{THz}$ devices and applications. Electrically controlled active metamaterials have their own indispensable potentials, and numerous electrically enabled metamaterials have been successfully demonstrated in microwave and millimeter wave bands [32-34]. However, in $\mathrm{THz}$ frequency range, straightforward copying such technologies is limited by device sizes and fabrication resolutions. Therefore, most reported electrically active THz metamaterials are combined with two-dimensional materials, borrowing their capabilities as transparent electrodes and electrical regulation of conductivity. Exemplified by graphenebased metamaterial, it can achieve a $100 \mathrm{GHz}$ modulation bandwidth at $1.5 \mathrm{THz}$ by applying an external voltage [35]. Another method is based on Schottky diodes. Schottky diodes were inset metamaterial unit gaps and the charge carrier concentration in the gaps were tuned by applying gate voltage to the two-dimensional electron gas in the Schottky diodes. With this method, Schottky diode controlled metamaterials can achieve 45\% modulation depth [36]. However, these voltage controlled metamaterials are limited either by the non-standard material systems, or low feasibilities in large-scale fabrication.

Embedding transistors [32, 33, 37] and other conventional integrated circuit process technologies [38] into metamaterials has been implemented in the microwave frequency band, and is beginning to expand to the $\mathrm{THz}$ region. Meanwhile, advanced complementary metaloxide-semiconductor (CMOS) technology has been used above $400 \mathrm{GHz}$ for the oscillator [39] and imaging applications [9]. The CMOS processes can fully meet the design requirements of metamaterials, even the design of 3D metamaterials can be realized by using multilayer metal processes [40]. The critical dimensions of the advanced CMOS back-end-of-line interconnections also fulfills the metamaterial geometry requirements for the THz applications. The front-end-of-line transistors and the back-end-of-line interconnections of the CMOS technology offer a path to control both the individual and array of metamaterials. To design and 
fabricate active metamaterials with controlling transistors monolithically with single CMOS technology is a potential solution for a large-scale $\mathrm{THz}$ modulator. The back-end-of-line metamaterial array and sub-arrays can be grouped for a variety of $\mathrm{THz}$ sub-bands with respective geometries, and can be actively controlled with the underlying CMOS transistors.

In this work, we demonstrated a CMOS based actively tunable $\mathrm{THz}$ metamaterial array (C-ATTMA) with its source and drain of a metal-oxide-semiconductor field effect transistor (MOSFET) connected to the metamaterial unit gaps. By electrically controlling the voltage applied to the transistor gate, the resonant frequency and phase can be adjusted by changing the equivalent circuit performance of the C-ATTMA. As the applied voltage increased from $0 \mathrm{~V}$ to $1.8 \mathrm{~V}$, the resonance peak was red shifted by $35 \mathrm{GHz}$. The phase transition was also realized by electrically controlling the $\mathrm{THz}$ metamaterials. Our C-ATTMA may provide a new approach to realize active $\mathrm{THz}$ modulator for multi-band $\mathrm{THz}$ wireless communications, sensing and imaging applications, as well as active phase shift devices for $\mathrm{THz}$ beam-forming and beamsteering applications.

\section{Methods}

\section{Device Design and Fabrication}

The schematic diagram of the C-ATTMA is illustrated in Fig. 1. The whole system is divided into multiple regions, as shown in the top left inset of this layout. Different regions with different parameters enable to the feasibility of expanding modulation bandwidth. Here, the effect of one region is assessed in detail, and the principle of the remaining regions is equivalent. The spilt ring resonator (SRR) array was fabricated on the high resistance silicon based on the CMOS process, and the operating range was limited to the $\mathrm{THz}$ band by strict parameter design. When the electric field of the plane wave was incident perpendicular to the gap, the LC resonance response appeared in the transmission spectrum. The inset on the bottom left illustrates the details of each unit. A transistor was embedded into the SRR by connecting the 
source and drain to the gap of the SRR. A DC control voltage was applied to the gate. When the transistor was turned off, the entire device exhibited passive metamaterial properties. As the control voltage increased from $0 \mathrm{~V}$ to $1.8 \mathrm{~V}$, the variation of the resonant frequency $f_{x}$ occur due to the effect of MOSFET.

The idea was achieved in a $180 \mathrm{~nm}$ six-layer metal process from Semiconductor Manufacturing International Corporation (SMIC), and the cross section of the process is exhibited in Fig. 2a. The metamaterial array was made on the sixth layer of metals, which was connected to the bottom transistor by applying vias from top metal to the bottom silicon. The transistor was fabricated by heavily doping two high-concentration n-zone on a p-type silicon substrate. The metamaterial contained a $23 \times 23$ periodic array of closely placed SRRs. The scanning electron microscope image of one unit with the size of $100 \mu \mathrm{m} \times 100 \mu \mathrm{m}$ is exhibited in Fig. 2b. The control principle of the metamaterial array is depicted in Fig. 2c. The whole system was supported by an external voltage $V_{c c}$ of $3.3 \mathrm{~V}$. A control voltage $V_{\text {control }}$ was applied to all of the transistors by connecting the gates to the external electrode. The whole metamaterial array can reach an area of $2.35 \mathrm{~mm} \times 2.35 \mathrm{~mm}$. In order to apply the control voltage effectively, it is necessary to package the device. In this case, the electrode was bonding to the printed circuit board (model: FR-4) with gold wire. The surface of the printed circuit board was applied by a gold deposition process to ensure the success of the bonding and the silica gel (LOCTITE ABLESTIK 2025D) was covered to protect the gold wire, as shown in Fig. 2d.

\section{Regulation Theoretical Analysis}

The schematic diagram of a transistor embedding into the SRR is drew in Fig. 3a. The finite element analysis software integration package CST Microwave Studio was employed to simulate the $\mathrm{THz}$ wave. When $\mathrm{THz}$ electromagnetic waves illuminated onto SRR surface, it caused a small, high-frequency current in the loop. Fig. 3a depicts a complete circulating current, which proves that the SRR was operating in the LC resonant state at this frequency. The equivalent circuit model of the SRR embedded transistor [37] is given in the inset of Fig. 3b, 
on which the accurate circuit parameters and relevant mathematical relations are determined. The intrinsic resistance and equivalent inductance of the SRR loop can be expressed as $R_{m}$ and $L_{m}$, respectively. Loop resistance $R_{m}$ can be calculated by the size of SRRs and aluminum resistivity. The equivalent inductance $L_{m}$ can be obtained by Equation (1) [41].

$$
L_{\mathrm{m}}=\frac{\lambda_{0} \mu_{0}}{5 \pi}\left(2.3 \log _{10}\left(\frac{8 \lambda_{0}}{5 d}\right)-2.85\right) \text {, }
$$

where $\lambda_{0}$ can be obtained by the dimension of SRRs, $\lambda_{0}=80 \mu \mathrm{m} \times 10$, and the final equivalent inductance $L_{m}$ is $\sim 2 \times 10^{-9} \mathrm{H}$. When the current passed through the gap, it would flow between source and drain in the transistor. The transistor can be seen as a parallel combination of total drain-to-source capacitance $\left(C_{d s}\right)$ and equivalent resistance $\left(R_{t}\right)$ between source and drain. In the transistors, $C_{d s}$ can be calculated from the known structural capacitance of the transistor [37] as shown in Equation (2), and would not be significantly varied when $V_{g s}$ was adjusted.

$$
C_{d s}=C_{d b}+\frac{C_{g d} C_{g s}}{C_{g d}+C_{g s}}
$$

For the $C_{g s}$ and $C_{g d}$ were negligible, $C_{d b}$ described the equivalent capacitance of MOSFET, which is $\sim 10^{-16} \mathrm{~F} . R_{t}$ is a variable resistor in the gap, which is originated from the equivalent channel resistance between the source and drain that varies dynamically with gate voltage $V_{\text {control. }}$ As the $V_{\text {control }}$ increased, the equivalent resistance $R_{t}$ was decreased. When the $V_{\text {control }}$ reached $1.8 \mathrm{~V}$, the equivalent resistance was minimum. For the equivalent impedance of $C_{t}$ ( $\left.\left(j \omega C_{t}\right)^{-1}\right)$ in the THz range was close to $R_{t}$, either one of them was not negligible in the calculation. On the other hand, since the RF current was a small AC current, the AC voltage $v_{d s}$ was so small that the conducting channel could not become "pinched-off". Based on the above conditions, the construction of equivalent circuit was considered to be believable.

Based on the constructed equivalent circuit model, the resonant frequency was firstly analyzed. As $R_{t}$ changes dynamically with the $V_{\text {control }}$, the resonant frequency would also change accordingly. Through the circuit model and known parameters, the resonant frequency $\omega_{0}$ of 
LC resonance system can be available from the basic definition of circuit resonance. Firstly, the total impedance $Z$ of the circuit can be obtained easily by the circuit model in Equation (3). From this Equation, the resonance frequency expression was obtained under the condition that the imaginary part of $Z$ was zero as shown in Equation (4).

$$
\begin{gathered}
Z=R_{m}+j \omega L_{m}+\frac{R_{t}}{1+j \omega C_{t} R_{t}}, \\
\omega_{0}=\sqrt{\frac{1}{C_{t} L_{m}}-\frac{1}{C_{t}^{2} R_{t}^{2}}},
\end{gathered}
$$

The curve of resonance frequency $f_{0}$ with resistance $R_{t}$ was obtained from the equation (4), as shown in Fig. 3b, we find that when $R_{t}$ exceeds $30 \mathrm{k} \Omega$, the slope is nearly flat. It is known that when the channel reaches its maximum width, the minimum $R_{t}$ can be achieved close to several thousand ohms. Here, we took $R_{t}$ from $5 \mathrm{k} \Omega$ to $40 \mathrm{k} \Omega$ to explore. The quality factor of the loop is defined as the ratio of energy stored to energy consumed. The inductance $L_{m}$ stores the magnetic energy in the resonant frequency, and the impedance $R_{m}$ describes the resistance of SRR. In fact, capacitance and inductance store the same energy in resonance, the expression can be further simplified according to this principle in Equation (5). On the other hand, due to the use of M6 metal layer to manufacture SRR structure, the material is aluminum with low resistivity. In the case of micron-level metamaterial structure, the resistance value of $R_{m}$ was still negligible compared with the transistor resistance $R_{t}$, even the transistor was in the conduction state.

$$
Q=\omega_{0} \frac{\frac{1}{4}\left|I_{L m}\right|^{2} L_{m}+\frac{1}{4}\left|V_{C_{t}}\right|^{2} C_{t}}{\frac{1}{2}\left|I_{R_{m}}\right|^{2} R_{m}+\frac{1}{2}\left|I_{R_{t}}\right|^{2} R_{t}}=\frac{\omega_{0} L_{m}\left(\omega_{0} C_{t} R_{t}\right)^{2}+1}{R_{t}},
$$

The ability to achieve negative permeability was considered an important property of SRRs. When LC resonance occurs, the permeability can be negative. Here, the expression of the permeability changing with resonance frequency is given in Equation (6) [37]. 


$$
\begin{gathered}
\mu_{r} \approx 1+\frac{F \omega^{2}}{\omega_{0}^{2}-\omega^{2}+\frac{j \omega \omega_{0}}{Q},} \\
F=\frac{\mu_{0} A_{\text {loop }}^{2}}{V_{\text {cell } L \text { loop }}},
\end{gathered}
$$

$F$ is the geometric factor of the unit cell, $A$ is the area contained in the SRR ring, and $V$ is the volume of the cell. Here, the $R_{t}$ values in the range of $5 \mathrm{k} \Omega$ to $30 \mathrm{k} \Omega$ were taken, and the corresponding permeability was calculated. The curves of the real and imaginary parts of the permeability varying with resistance were obtained indirectly in Fig. 4a and Fig. 4b. It was found that the permeability varied with the resistance in a strict rule. Then the transmission coefficient can be further calculated in Equation (7) according to the above parameters [42].

$$
T^{-1}=\left[\cos (n k d)-\frac{i}{2}\left(z+\frac{1}{z}\right) \sin (n k d)\right] e^{i k d}
$$

where $n$ is the refractive index $\left(n=\sqrt{\mu_{r} \varepsilon_{r}}\right), z$ is the impedance $\left(z=\sqrt{\mu_{r} / \varepsilon_{r}}\right)$, and the dielectric constant of LC resonance is a positive number assumed to be $1, d$ is the length of each cell, and $k=\omega / c$ is the wavenumber of the incident wave. The variation of the calculated transmission parameters with the transistor resistance $R_{t}$ is plotted in Fig. $4 \mathrm{c}$, as the resistance increases, the resonant frequency produces a blue shift.

\section{Results and Discussion}

The THz experiments were performed in a home-built $\mathrm{THz}$ time-domain spectrometer driven by a commercial Ti: sapphire laser oscillator. The center wavelength of the femtosecond laser pulses was $800 \mathrm{~nm}$, the pulse duration was $70 \mathrm{fs}$, and the repetition frequency was $80 \mathrm{MHz}$. By dividing the laser beam into two beams, $90 \%$ of the energy was used to excite the GaAs photoconductive antenna with a $50 \mu \mathrm{m}$ gap (Z-omega), and the maximum pumping effect focused on the transmitter was $\sim 3 \mu \mathrm{J} / \mathrm{cm}^{2}$. The residual energy was used to detect THz signals 
in a ZnTe detector with a thickness of $1 \mathrm{~mm}$. All the measurements were carried out under dry nitrogen gas environment to eliminate the fluence from water vapor.

Firstly, to describe the effect of $180 \mathrm{~nm}$ CMOS process on the metamaterial, a single-layer SRRs array without embedded transistor was designed as a control sample. In order to study its transmission performance, plane $\mathrm{THz}$ waves with an electric field perpendicular to the gap was applied. Fig. 5a illustrates the experimental and simulation results of the single-layer SRRs. It is found that LC resonance and plasmon resonance were generated $\sim 0.3 \mathrm{THz}$ and $0.9 \mathrm{THz}$, manifesting that the $\mathrm{THz}$ metamaterials were successfully realized by using CMOS process. Fig. 5b exhibits the experimental and simulation results of a double-layer SRR structure, which adopted the same structure parameters as that from the single-layer SRRs. The two openings above were staggered and connected by a via, which is extremely difficult when just using the lithography and evaporation procedures. The device had no transistors. The experimental and simulation results showed that the LC resonance strength in double layer ring structures is obviously weakened. It is speculated that the interaction between the two layers at this time affects the LC resonance effect of metamaterials.

Then the SRR array embedded with transistors was repeatedly tested to obtain the frequency domain waveform affected by different voltage. Fig. 6a depicts the test results with varying resonance frequencies from $0.25 \mathrm{THz}$ to $0.36 \mathrm{THz}$ when applied various control voltage $V_{\text {control. }}$ Unfortunately, there was no significant LC resonance in the expected range, for the external conditions such as the effects of the printed circuit board and the silica gel may have interfered with the results. To verify this speculation, bare sample without packaging was tested, and the results are shown in the inset of Fig. 6a. The apparent LC resonance was obtained at $0.32 \mathrm{THz}$. In order to reflect the effect of applied $V_{\text {control }}$ on the experimental results, the measured data under different $V_{\text {control }}$ was normalized, which is plotted in Fig. $6 \mathrm{~b}$. The data were obtained from dividing the experimental results with different $V_{\text {control }}$ values by the result from 
$0 \mathrm{~V}$, respectively. It is found that the waveform changes significantly with the $V_{\text {control}}$, indicating the reliability of this measurement.

To intuitively extract the electrically induced modulation phenomenon, an equivalent transformation was introduced. The measured data of the unpackaged bare chip in the inset of Fig. 6a can be considered as the result for $0 \mathrm{~V}$ applied $V_{\text {control }}$. Fig. $6 \mathrm{c}$ is the processed results obtained by multiplying the test data of the unpackaged bare chip with the normalized value of Fig. 6b. The resonance frequency variation was obtained by multiplying the two signals together to amplify the modulation trend. This method overcomes the influence of packaging interference and the final signal shows an obvious red shift along with the gradual increasing of the applied voltages. Fig. 6d illustrates the relative frequency variation as a function of the external applied voltages, further visually corroborating the red shift phenomenon. When the $V_{\text {control }}$ exceeded $0.6 \mathrm{~V}$, the redshift trend was found to be slowed down. This behavior can be attributed to the intrinsic characteristics of channel resistances under gate voltages. Since the $V_{\text {control }}$ was ultimately applied to the gate, the increase of voltage meant the decrease of $R_{t}$. When the $V_{\text {control }}$ was below the threshold voltage $V_{t}$, the transistor was working in the cut-off region, and the resistance dropped rapidly with the increase of the voltage. However, when the applied voltage exceeded the $V_{t}$, the conductive channel was almost formed. In this case, the transistor was working in the linear region, and the resistance no longer promptly decreased with the increase of the voltage. As a consequence, our experimental results have already been quantitatively reproduced by the calculated results.

For the effect of gate voltage on phase variation, it was also comprehensively evaluated, and the phase difference from $0.25 \mathrm{THz}$ to $0.36 \mathrm{THz}$ is plotted in Fig. 6e. Obviously seen from this figure, the phase differences are varied for different voltages. The summarized results are exhibited in Fig. 6f. It can be found that the phase difference decreased with the increase of the applied voltage, and the maximum absolute phase difference reached up to $3^{\circ}$. Although the phase tunability was weak in this principle verification experiments, our preliminary results 
open the door of CMOS compatible electrically controlled phase devices and systems. We believe that with more delicate design and manufacturing, the future phase sensitive $\mathrm{THz}$ metamaterials regulated by electricity can be fabricated soon, and will play an important role in large-scale applications.

\section{Conclusion}

In summary, CMOS based actively tunable THz metamaterial arrays (C-ATTMA) with split ring resonators (SRRs) have been successfully demonstrated, which performed as a tunable device at $0.3 \mathrm{THz}$. The experimental results show that the resonance frequency can be redshifted by $35 \mathrm{GHz}$ and the phase can be engineered along with the increase of the applied bias voltage from $0 \mathrm{~V}$ to $1.8 \mathrm{~V}$. The preliminary experimental results can be well interpreted by equivalent circuit model. We believe that the CMOS technology has great potential in making controllable THz metamaterials, which can be easily applied at frequencies above $200 \mathrm{GHz}$ through scaling in size. This work can be further extended to the programmable and encrypted intelligent $\mathrm{THz}$ metamaterials, enabled by proposed electrically active THz units. The C-ATTMA pave the way to a range of applications in the imaging, sensing and wireless communications field.

\section{Abbreviations}

CMOS: Complementary metal-oxide-semiconductor; C-ATTMA: CMOS based actively tunable $\mathrm{THz}$ metamaterial array; SRR: Split ring resonator; MOSFET: metal-oxidesemiconductor field effect transistor.

\section{Availability of data and materials}

The datasets used and/or analysed during the current study are available from the corresponding author on reasonable request. 


\section{Conflicts of interest}

The authors declare that there are no conflicts of interest related to this article.

\section{Funding}

National Key R\&D Program of China (No. 2018YFB0407602), National Natural Science Foundation of China $(61774013,11827807,61905007)$, Beijing Natural Science Foundation (4194083).

\section{Acknowledgements}

This study is also supported by the "Zhuoyue Program" of Beihang University (ZG216S18B5), Qingdao Innovation and Entrepreneurship Leadership Program (18-1-2-21-zhc), and the VR innovation platform from Qingdao Science and Technology Commission and Magnetic Sensor innovation platform from LaoShan District.

\section{Authors' contributions}

Prof. L. G. Wen, Prof. T. X. Nie and Prof. X. J. Wu conceived original idea of combining CMOS technology with metamaterials. Y. S. Liu and Z. Y. Bai presented the operation mode of metamaterials. T. Sun designed and made the external circuit. Y. S. Liu and T. Sun conducted experiments. Y. Sun and H. L. Li played an important role in experiment and simulation. Prof. X. J. Wu, Prof. T. X. Nie and Prof. Y. Hu provided theoretical guidance and technical support. Prof. C. J. Ruan, H. Y. Zhang, K. L. Chen and Y. Z. Sun provided valuable advice and all the authors contributed to the writing. Prof. T. X. Nie and Prof. X. J. Wu supervised the project. The authors read and approved the final manuscript. 


\section{References:}

1. S. Keren-Zur, M. Tal, S. Fleischer, D. M. Mittleman, T. Ellenbogen. Generation of spatiotemporally tailored terahertz wavepackets by nonlinear metasurfaces. Nat Commun. 2019;10.

2. A. Y. Pawar, D. D. Sonawane, K. B. Erande, D. V. Derle. Terahertz technology and its applications. Drug Invention Today. 2013;5:157-163.

3. K. Serita, E. Matsuda, K. Okada, H. Murakami, I. Kawayama, M. Tonouchi. Invited Article: Terahertz microfluidic chips sensitivity-enhanced with a few arrays of meta-atoms. APL Photonics. 2018;3:51603.

4. M. Tonouchi. Cutting-edge terahertz technology. Nat Photonics. 2007;1: 97-105.

5. T. Kleine-Ostmann, T. Nagatsuma. A Review on Terahertz Communications Research. Journal of Infrared, Millimeter, and Terahertz Waves. 2011;32:143-171.

6. X. Wu, B. Quan, X. Pan, X. Xu, X. Lu, C. Gu, L. Wang. Alkanethiol-functionalized terahertz metamaterial as label-free, highly-sensitive and specificbiosensor. Biosensors and Bioelectronics. 2013;42:626-631.

7. X. Yan, M. Yang, Z. Zhang, L. Liang, D. Wei, M. Wang, M. Zhang, T. Wang, L. Liu, J. Xie, J. Yao. The terahertz electromagnetically induced transparency-like metamaterials for sensitive biosensors in the detection of cancer cells. Biosens Bioelectron. 2018;126:485.

8. X. Yang, X. Zhao, K. Yang, Y. Liu, Y. Liu, W. Fu, Y. Luo. Biomedical Applications of Terahertz Spectroscopy and Imaging. Trends Biotechnol. 2016;34:810-824.

9. E. Ojefors, U. R. Pfeiffer, A. Lisauskas, H. G. Roskos. A 0.65 THz Focal-Plane Array in a Quarter-Micron CMOS Process Technology. IEEE J Solid-St Circ. 2009;44:1968-1976.

10. C. Sirtori, S. Barbieri, R. Colombelli. Wave engineering with THz quantum cascade lasers. Nat Photonics. 2013;7:691-701.

11. A. Forrer, L. Bosco, M. Beck, J. Faist, G. Scalari. RF Injection of THz QCL Combs at 80 K Emitting over 700 GHz Spectral Bandwidth. Photonics. 2020;7:9.

12. T. Ishibashi, Y. Muramoto, T. Yoshimatsu, H. Ito. Unitraveling-Carrier Photodiodes for Terahertz Applications. IEEE J Sel Top Quant. 2014;20:79-88.

13. K. Liu, S. Jia, S. Wang, X. Pang, W. Li, S. Zheng, H. Chi, X. Jin, X. Zhang, X. Yu. 100 Gbit/s THz Photonic Wireless Transmission in the 350-GHz Band With Extended Reach. IEEE Photonic Tech L. 2018;30:10641067.

14. D. Kong, X. Wu, B. Wang, T. Nie, M. Xiao, C. Pandey, Y. Gao, L. Wen, W. Zhao, C. Ruan, J. Miao, Y. Li, L. Wang. Broadband Spintronic Terahertz Emitter with Magnetic - Field Manipulated Polarizations. Adv Opt 
Mater. 2019;1900487.

15. T. Seifert, S. Jaiswal, U. Martens, J. Hannegan, L. Braun, P. Maldonado, F. Freimuth, A. Kronenberg, J. Henrizi, I. Radu, E. Beaurepaire, Y. Mokrousov, P. M. Oppeneer, M. Jourdan, G. Jakob, D. Turchinovich, L. M. Hayden, M. Wolf, M. Münzenberg, M. Kläui, T. Kampfrath. Efficient metallic spintronic emitters of ultrabroadband terahertz radiation. Nat Photonics. 2016;10:483-488.

16. X. Chen, X. Wu, S. Shan, F. Guo, D. Kong, C. Wang, T. Nie, C. Pandey, L. Wen, W. Zhao, C. Ruan, J. Miao, Y. Li, L. Wang. Generation and manipulation of chiral broadband terahertz waves from cascade spintronic terahertz emitters. Appl Phys Lett. 2019;115:221104.

17. B. Wang, S. Shan, X. Wu, C. Wang, C. Pandey, T. Nie, W. Zhao, Y. Li, J. Miao, L. Wang. Picosecond nonlinear spintronic dynamics investigated by terahertz emission spectroscopy. Appl Phys Lett. 2019;115:121104.

18. Z. Fang, H. Wang, X. Wu, S. Shan, C. Wang, H. Zhao, C. Xia, T. Nie, J. Miao, C. Zhang, W. Zhao, L. Wang. Nonlinear terahertz emission in the three-dimensional topological insulator $\mathrm{Bi}_{2} \mathrm{Te}_{3}$ by terahertz emission spectroscopy. Appl Phys Lett. 2019;115:191102.

19. Chen H, Su J, Wang J, Zhao X. Optically-controlled high-speed terahertz wave modulator based on nonlinear photonic crystals. Optics express. 2011;19(4): 3599-3603.

20. Cheng L, Jin Z, Ma Z, Su F, Zhao Y, Zhang Y, Su T, Sun Y, Xu X, Ming Z, Bian Y, Sheng Z. Mechanical Terahertz Modulation Based on Single-Layered Graphene. Advanced Optical Materials. 2018;6(7):1700877.

21. Gwin A H, Kodama C H, Laurvick T V, Coutu R. A, Jr, Taday P. F. Improved terahertz modulation using germanium telluride (GeTe) chalcogenide thin films. Applied Physics Letters. 2015;107(3): 031904.

22. M. M. Jadidi, A. B. Sushkov, R. L. Myers-Ward, A. K. Boyd, K. M. Daniels, D. K. Gaskill, M. S. Fuhrer, H. D. Drew, T. E. Murphy. Tunable Terahertz Hybrid Metal-Graphene Plasmons. Nano Lett. 2015;15:7099-7104.

23. Y. Jeong, J. Kyoung, J. Choi, S. Han, H. Park, N. Park, B. Kim, H. Kim, D. Kim. Terahertz nano antenna enabled early transition in $\mathrm{VO}_{2}$. Nano Lett. 2012.

24. H. Chen, M. J. Cich, W. J. Padilla, R. D. Averitt, A. K. Azad, A. J. Taylor. A metamaterial solid-state terahertz phase modulator. Nat Photonics. 2009;3:148-151.

25. W. Cao, R. Singh, I. A. Al-Naib, M. He, A. J. Taylor, W. Zhang. Low-loss ultra-high-Q dark mode plasmonic Fano metamaterials. Opt Lett. 2012;37:3366-3368.

26. R. Singh, I. A. I. Al-Naib, M. Koch, W. Zhang. Sharp Fano resonances in THz metamaterials. Opt Express. 2011;19:6312.

27. M. Gupta, V. Savinov, N. Xu, L. Cong, G. Dayal, S. Wang, W. Zhang, N. I. Zheludev, R. Singh. Sharp Toroidal 
Resonances in Planar Terahertz Metasurfaces. Adv Mater. 2016;28:8206-8211.

28. X. Zhang, Y. Lin. Actively electromagnetic modulation of IHI-shaped terahertz metamaterial with highefficiency switching characteristic. Results Phys. 2019;15:102532.

29. Z. Yangjian, F. Chuhuan, L. Qi, S. Xin, Y. Hongbin. Dynamic Reflection Phase Modulation in Terahertz Metamaterial. IEEE Photonics. 2019;11:1-12.

30. H. Chen, W. J. Padilla, J. M. O. Zide, A. C. Gossard, A. J. Taylor, R. D. Averitt. Active terahertz metamaterial devices. Nature. 2006;444:597-600.

31. W. J. Padilla, A. J. Taylor, C. Highstrete, M. Lee, R. D. Averitt. Dynamical electric and magnetic metamaterial response at terahertz frequencies. Phys Rev Lett. 2006;96:107401.

32. T. J. Cui, M. Q. Qi, X. Wan, J. Zhao, Q. Cheng. Coding metamaterials, digital metamaterials and programmable metamaterials. Light: Science \& Applications. 2014;3:e218.

33. L. Zhang, X. Q. Chen, S. Liu, Q. Zhang, J. Zhao, J. Y. Dai, G. D. Bai, X. Wan, Q. Cheng, G. Castaldi, V. Galdi, T. J. Cui. Space-time-coding digital metasurfaces. Nat Commun. 2018;9.

34. L. Li, T. Jun Cui, W. Ji, S. Liu, J. Ding, X. Wan, Y. Bo Li, M. Jiang, C. Qiu, S. Zhang. Electromagnetic reprogrammable coding-metasurface holograms. Nat Commun. 2017;8.

35. S. J. Kindness, N. W. Almond, B. Wei, R. Wallis, W. Michailow, V. S. Kamboj, P. Braeuninger-Weimer, S. Hofmann, H. E. Beere, D. A. Ritchie, R. Degl'Innocenti. Active Control of Electromagnetically Induced Transparency in a Terahertz Metamaterial Array with Graphene for Continuous Resonance Frequency Tuning. Adv Opt Mater. 2018;6:1800570.

36. M. T. Nouman, H. Kim, J. M. Woo, J. H. Hwang, D. Kim, J. Jang. Terahertz Modulator based on Metamaterials integrated with Metal-Semiconductor-Metal Varactors. Sci Rep-UK. 2016;6:26452.

37. J. P. Barrett, A. R. Katko, S. A. Cummer. Bandwidth Tuning in Transistor Embedded Metamaterials Using Variable resistance. Progress In Electromagnetics Research. 2016;157:49-61.

38. M. Manjappa, P. Pitchappa, N. Singh, N. Wang, N. I. Zheludev, C. Lee, R. Singh. Reconfigurable MEMS Fano metasurfaces with multiple-input - output states for logic operations at terahertz frequencies. Nat Commun. 2018;9.

39. C. C. D. S. Eunyoung Seok, C. H. K. K. David B. Tanner. A 410GHz CMOS Push-Push Oscillator with an On-Chip Patch Antenna. in IEEE International Solid-State Circuits Conference San Francisco, 2008;472473.

40. I. Escorcia Carranza, J. P. Grant, J. Gough, D. Cumming. Terahertz Metamaterial Absorbers Implemented in CMOS Technology for Imaging Applications: Scaling to Large Format Focal Plane Arrays. IEEE J Sel Top 
Quant. 2017;23:1-8.

41. T. Hand, S. Cummer. Characterization of Tunable Metamaterial Elements Using MEMS Switches. IEEE Antenn Wirel Pr. 2007;6:401-404.

42. D. R. Smith, S. Schultz, P. Markoš, C. M. Soukoulis. Determination of effective permittivity and permeability of metamaterials from reflection and transmission coefficients. Phys Rev B. 2002;65. 


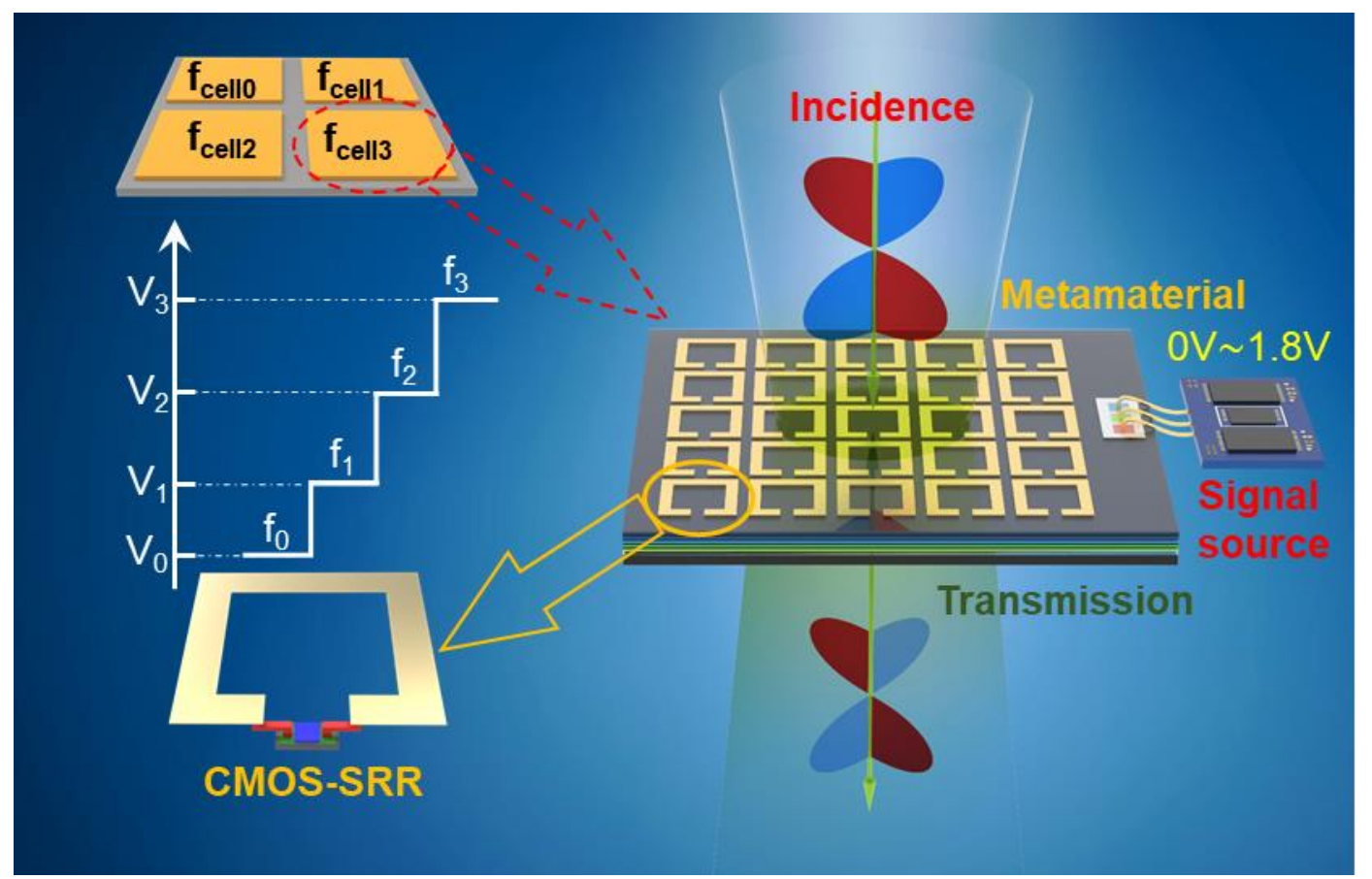

Fig. 1 Working principle of the CMOS compatible THz metamaterials. THz waves illuminate onto the upper side of the electronically controlled metamaterial cell, and the transmitted amplitudes and phases are modulated by the applied voltage signals. Cell arrays can be employed to realize more advanced electromagnetic wave regulation such as spatial light modulation, beamforming and beam steering devices, et cetera. 


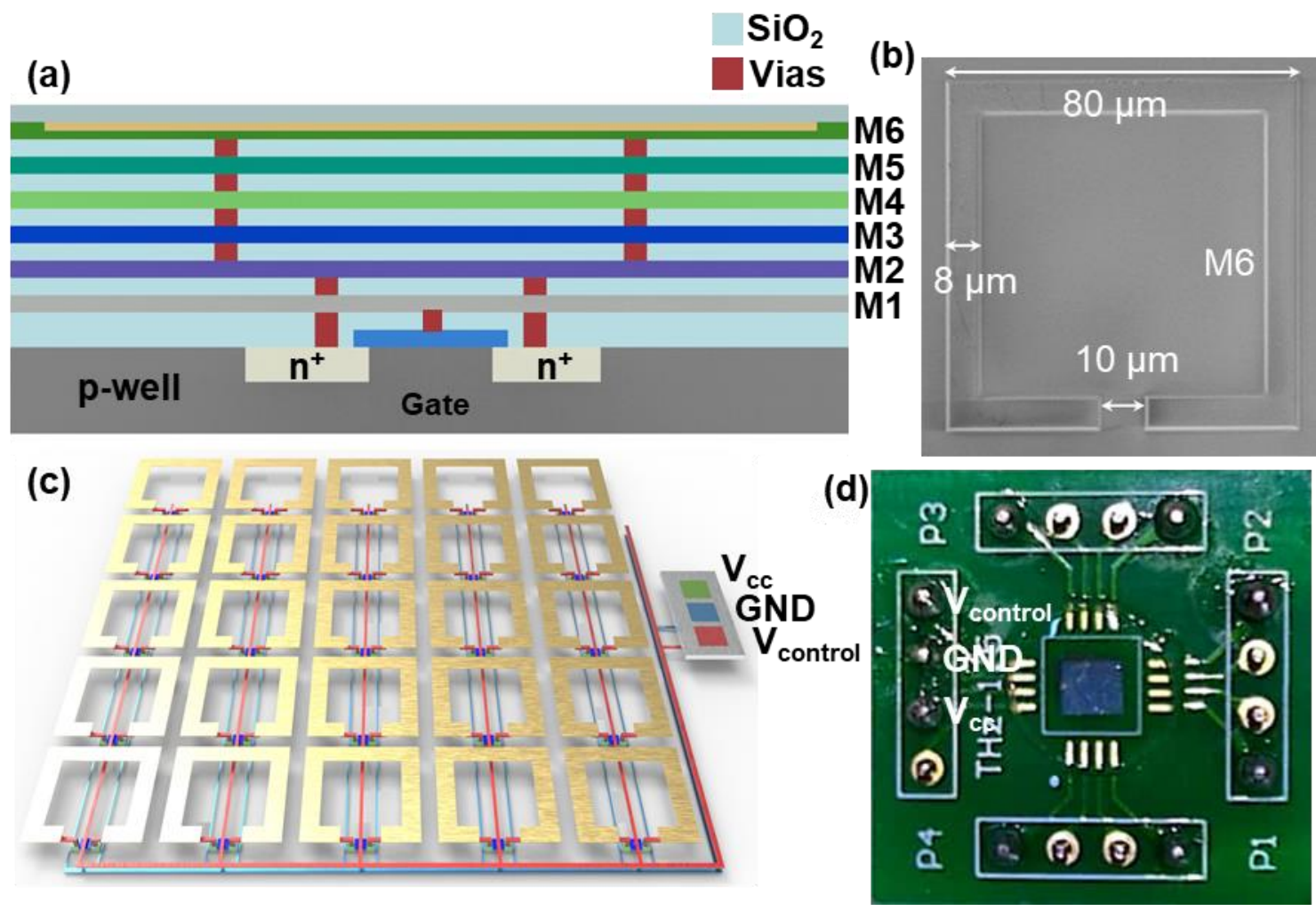

Fig. 2 Schematic diagram of the C-ATTMA fabrication and its photo. a) cross-sectional diagram of a single unit, in which M6 metal was used for the metamaterial structure, and M1 metal was used to control the line layout. b) The scanning electron microscopy image of one unit. The structural parameters are as follows. Unit period: $100 \mu \mathrm{m}$. Square dimension: $80 \mu \mathrm{m}$. Gap width: $10 \mu \mathrm{m}$. Line width: $8 \mu \mathrm{m}$. Al thickness: $200 \mathrm{~nm}$. c) Layout and wiring diagram of the C-ATTMA. d) Physical diagram of the device packaging and external circuit. The electrode was bonding to the printed circuit board with gold wire, and the surface was covered with silica gel to protect the gold wire. 
(a)

(b)

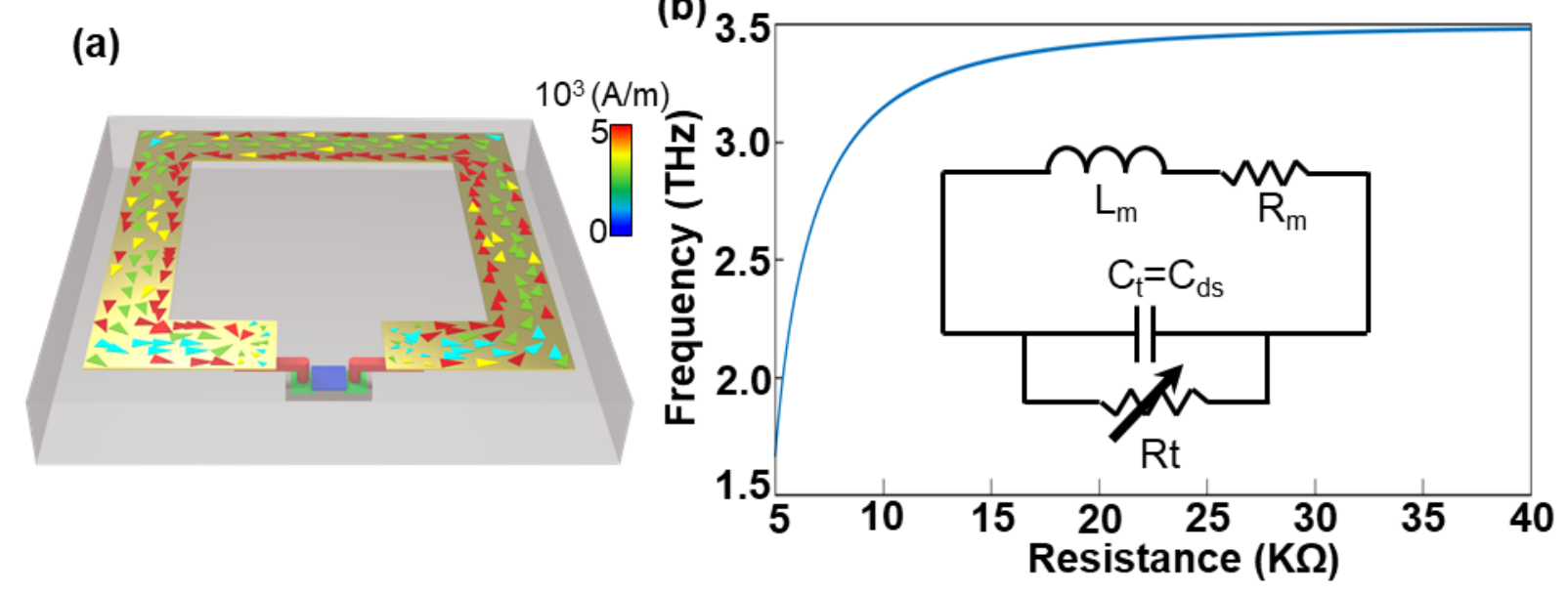

Fig. 3 Finite element simulation and numerical calculation based on LC equivalent model. a) The current flow of the SRRs at LC resonance frequency. b) The equivalent circuit model and the LC resonance frequency as a function of the SRR unit gap resistance. 

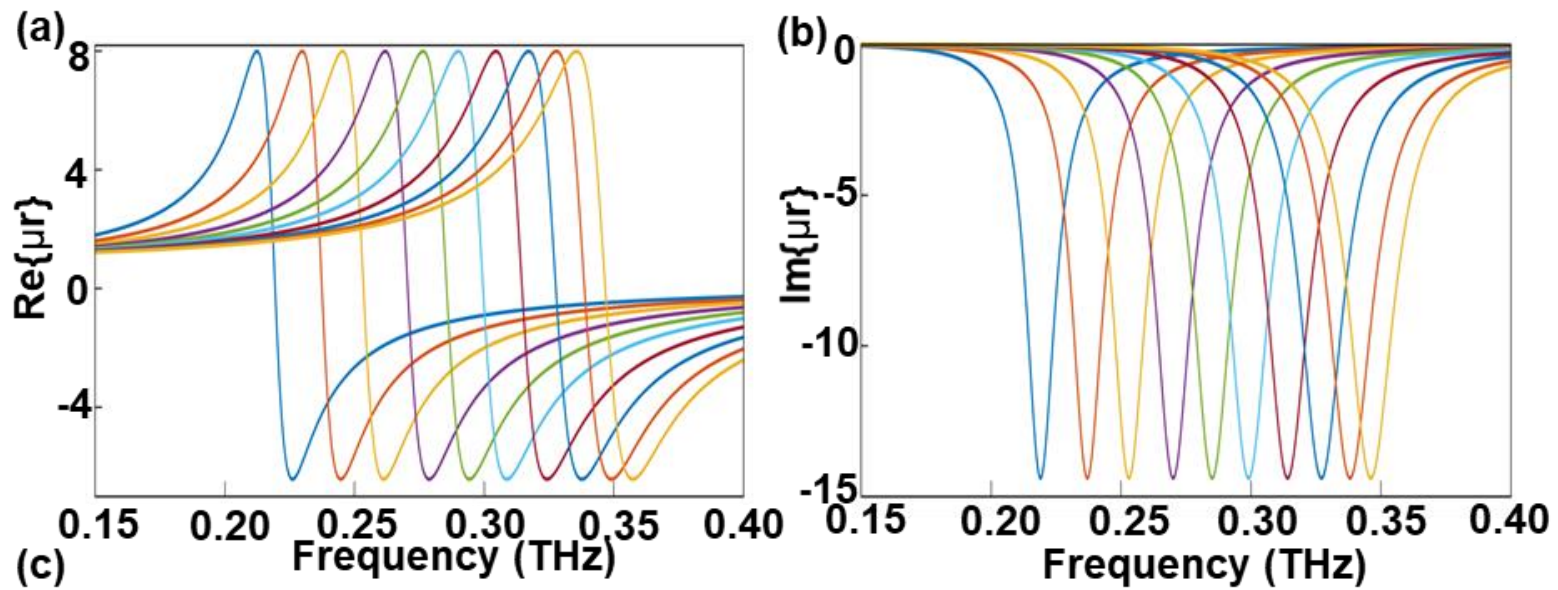

(c)
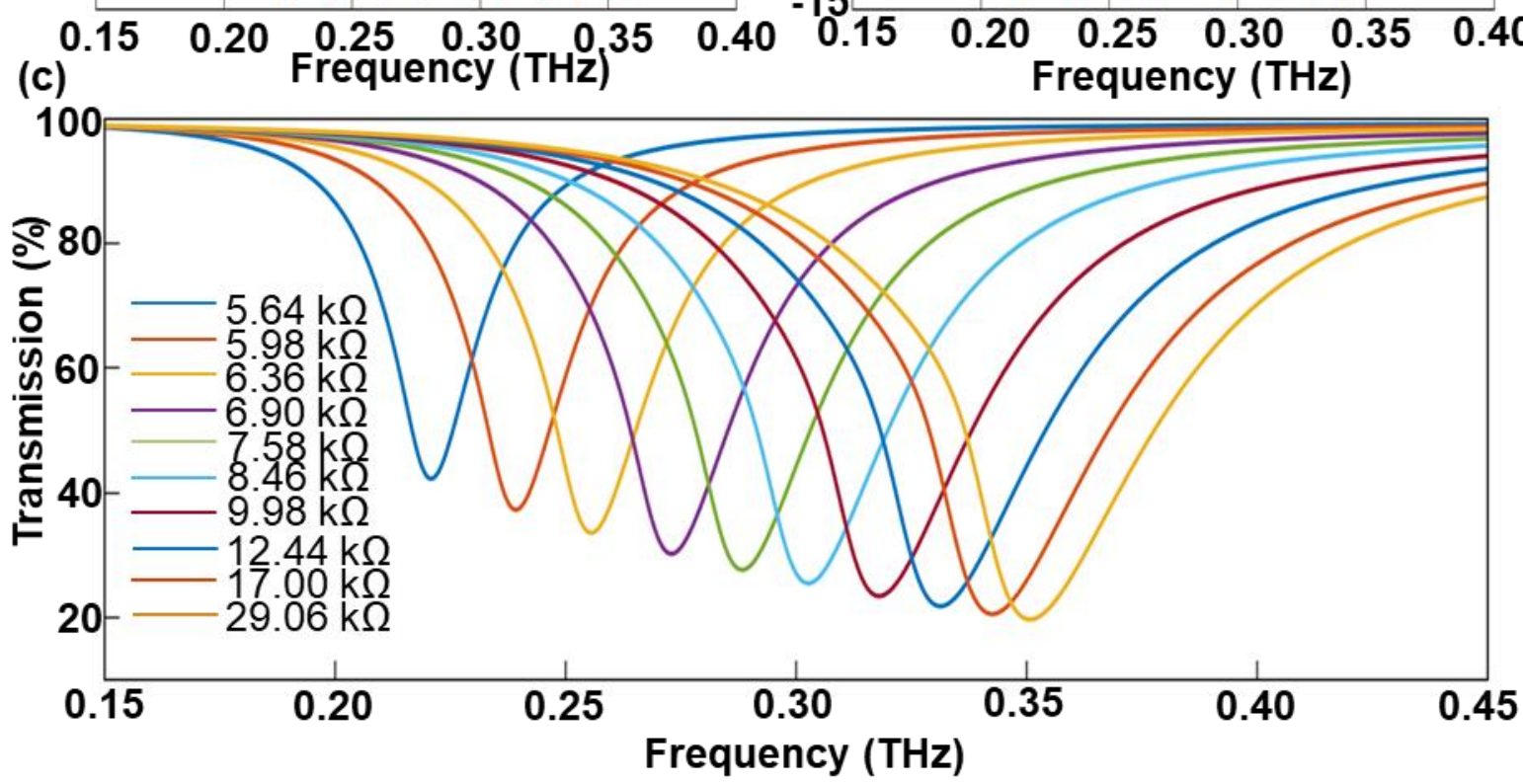

Fig. 4 The predicted C-ATTMA modulation performance under the calculated equivalent. a) $\left.\operatorname{Re}\left\{\mu_{\mathrm{r}}\right\}, \mathrm{b}\right) \operatorname{Im}\left\{\mu_{\mathrm{r}}\right\}$ and c) transmittance of the metamaterials by changing the $R_{\mathrm{t}}$. 

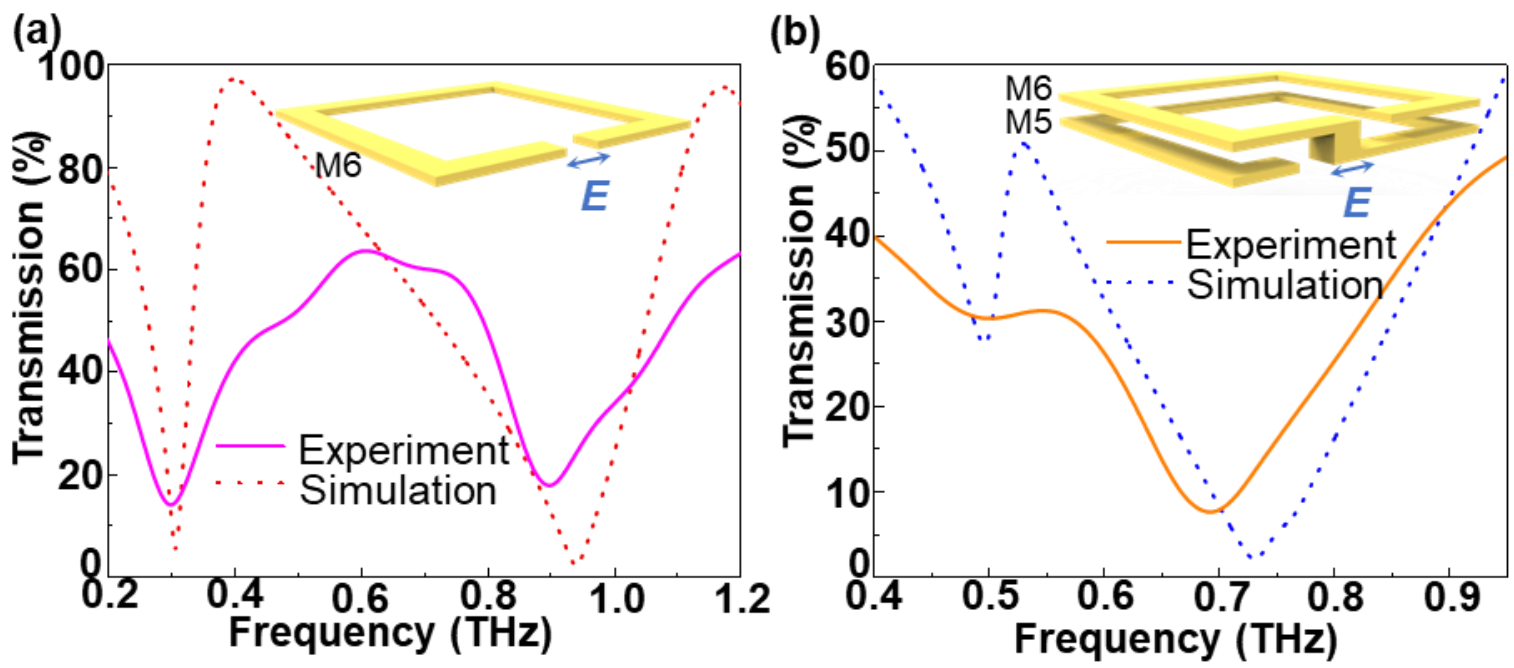

Fig. 5 Verification of CMOS enabled THz metamaterials. Simulation and experimental results for a) single-layer and b) double-layer open SRRs without MOSFET. Obvious resonances were probed, manifesting the feasibility of CMOS compatible THz metamaterials. 
(a)
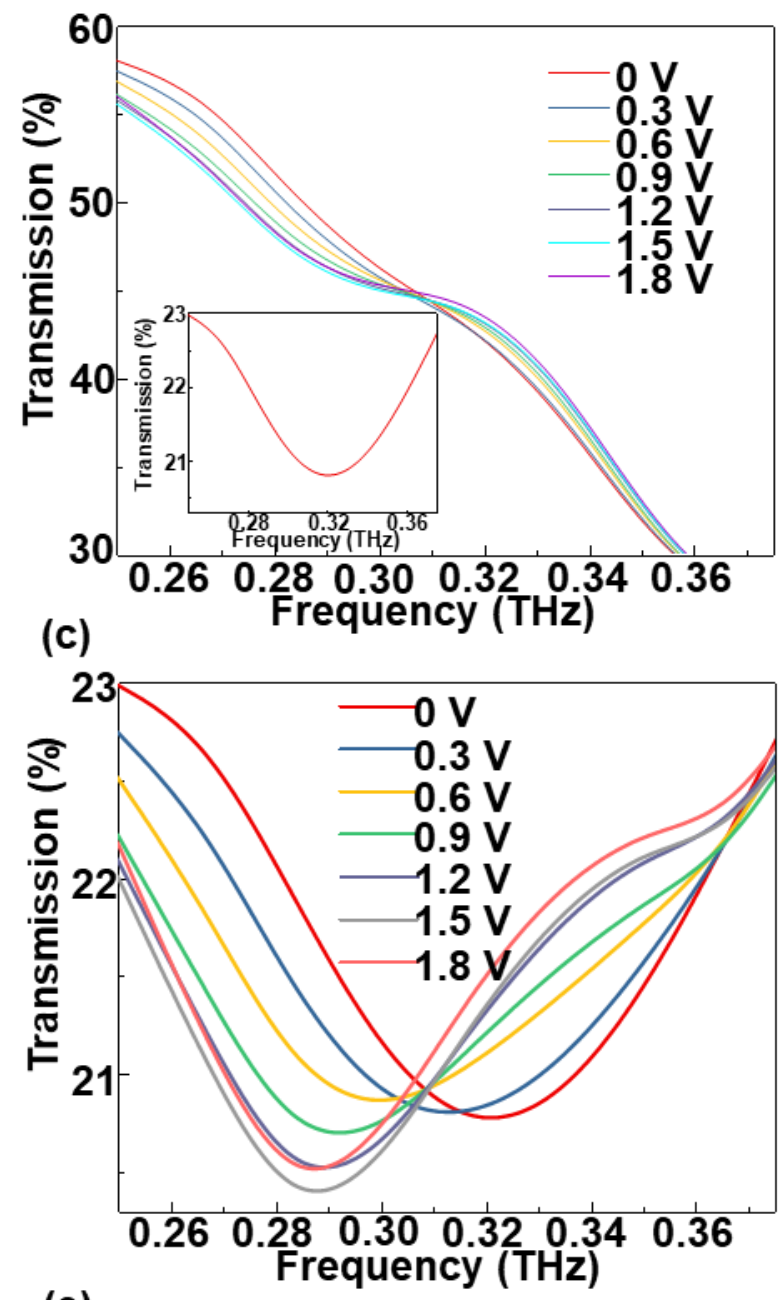

(e)

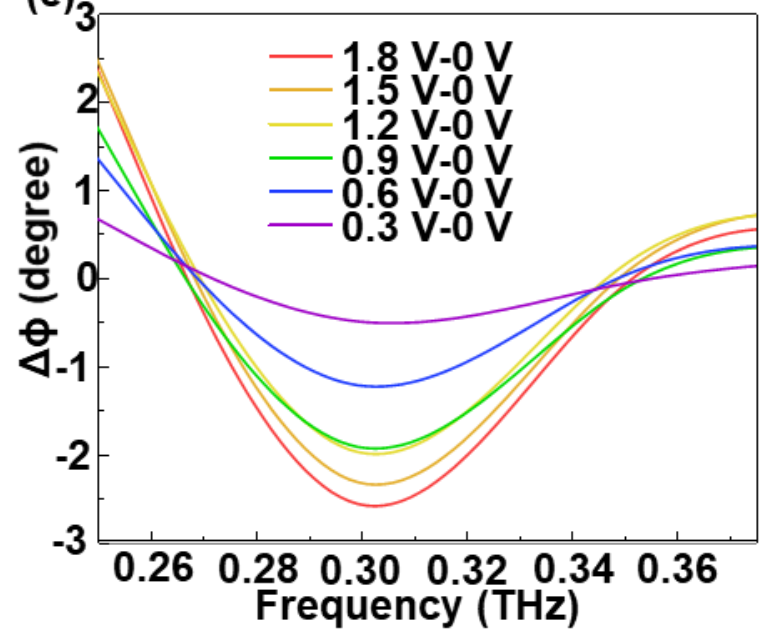

(b)
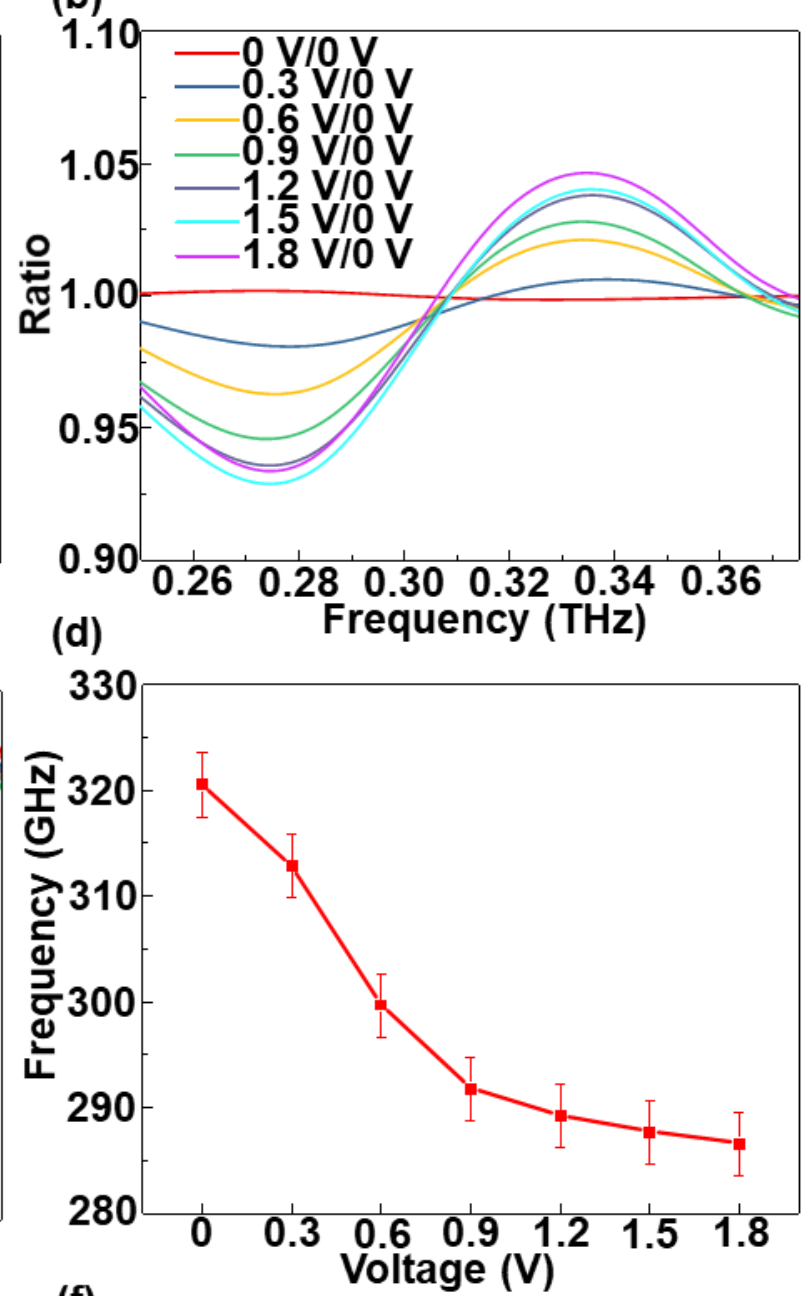

(f)

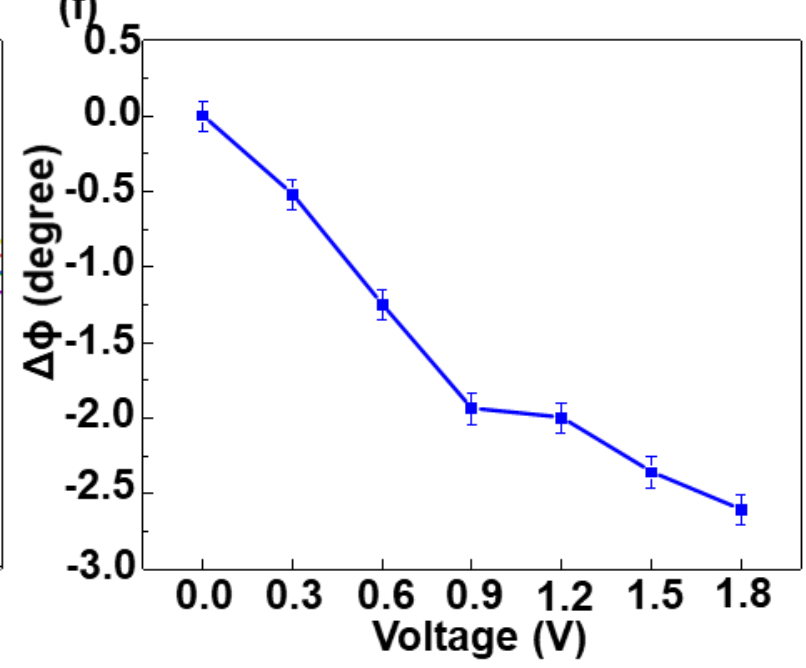

Fig. 6 Experimental results for the electrically engineered C-ATTMA device. a) Transmitted THz amplitude spectra of SRRs embedded with MOSFET with different applied voltages. b) The amplitude spectrum ratio for signals under different voltages divided by that from $0 \mathrm{~V}$. c) The extracted LC resonant amplitude spectra of different voltage. d) The corresponding resonant frequency as a function of the applied voltages. e) Phase tunability depends on the applied voltages, while f) the summarized phase difference variation trend. 